\title{
AN AUTONOMOUS GPS-DENIED UNMANNED VEHICLE PLATFORM BASED ON BINOCULAR VISION FOR PLANETARY EXPLORATION
}

\author{
Man Qin ${ }^{1,2, *}$, Xue Wan ${ }^{1,2}$, Yu-Yang Shao ${ }^{1,2}$, Sheng-Yang Li ${ }^{1,2}$ \\ ${ }^{1}$ Technology and Engineering Center for Space Utilization, Chinese Academy of Sciences, Beijing, China - (shyli, wanxue, shaoyy, \\ qinman)@ csu.ac.cn \\ ${ }^{2}$ Key Laboratory of Space Utilization, Chinese Academy of Sciences, Beijing, China - (shyli, wanxue, shaoyy, qinman)@ csu.ac.cn
}

KEY WORDS: Planetary Exploration, Unmanned Vehicle Platform, Vision-based Navigation, Feature Matching, 3D Reconstruction

\begin{abstract}
:
Vision-based navigation has become an attractive solution for autonomous navigation for planetary exploration. This paper presents our work of designing and building an autonomous vision-based GPS-denied unmanned vehicle and developing an ARFM (Adaptive Robust Feature Matching) based VO (Visual Odometry) software for its autonomous navigation. The hardware system is mainly composed of binocular stereo camera, a pan-and tilt, a master machine, a tracked chassis. And the ARFM-based VO software system contains four modules: camera calibration, ARFM-based 3D reconstruction, position and attitude calculation, BA (Bundle Adjustment) modules. Two VO experiments were carried out using both outdoor images from open dataset and indoor images captured by our vehicle, the results demonstrate that our vision-based unmanned vehicle is able to achieve autonomous localization and has the potential for future planetary exploration.
\end{abstract}

\section{INTRODUCTION}

Accurate and autonomous localization of spacecraft and rover is essential for planetary exploration. Traditional navigation method based on ground remote control system cannot achieve autonomous navigation in terms of communication latency and bandwidth limitation. While autonomous navigation can be achieved by GPS on Earth, there is no such positioning system on other planets. The optical camera has the characteristics of low cost, wide application range, low power consumption and large field of view compared with the other detection devices, thus vision-based navigation has become an attractive solution for autonomous navigation. The combination of unmanned vehicle platform and robust computer vision algorithms is a challenge task. In this paper, a binocular camera based unmanned vehicle platform has been built and an ARFM (Adaptive Robust Feature Matching) based VO (Visual Odometry) software system has been developed to achieve GPS-denied autonomous navigation for planetary exploration.

\section{RELATED WORK}

In 2004, NASA has launched Spirit and Opportunity (Cheng Y et al., 2006), after that, the Mars Science Laboratory (MSL) team has launched the Curiosity rover (Sumner D, 2013) in August 2012. In 2013, China has launched Yutu (Zhou J et al., 2014), which has accomplished the exploration mission until 2016. ESA will launch Exo Mars (Griffiths A D et al., 2006) in the near future. Vision-based navigation had been used for planetary exploration in these unmanned vehicles, which proved successfully. However, vision-based navigation for planetary rover has the following challenges: 1) The images taken may contain scale and rotation distortions; 2) The real-time and accuracy requirement must be met; 3 ) There are some featureless areas which may decrease the feature matching accuracy. Thus, the matching result of traditional feature extraction algorithms, such as Harris (Stephens M, 1988), Shi-Tomasi, Susan corner detection algorithm and SIFT (Scale Invariant Feature Transform) (David G. Lowe, 2004), are unsatisfied. Thus, real-time, accuracy and reliability must be taken into consideration, not only in hardware but also in software.

\section{THE BINOCULAR VISION BASED AUTONOMOUS UNMANNED VEHICLE}

In this paper, an unmanned vehicle platform based on binocular vision has been designed and built. We also developed an ARFM-based VO software for its autonomous navigation. This section will present our work in terms of both hardware system and the software system.

The unmanned vehicle platform can navigate by the ARFMbased VO software automatically in the process of walking, but manually stop or start is needed.

\subsection{The hardware system}

The unmanned vehicle we designed is a ground mobile robot with three independent hierarchical layers, as is shown in Fig. 1. The upper layer is made up of a binocular camera and a pan-and-tilt, which is used to receive the image sequence and precisely control the attitude of the camera. The middle layer is a master machine for processing the image streams transferred from the upper and issuing instructions, for example, moving forward or backward. The lower layer is a tracked chassis which can drive this unmanned vehicle according to the instructions received form the middle. The maximum speed is set to $0.1 \mathrm{~m} / \mathrm{s}$ because of the rough surface of planetary.

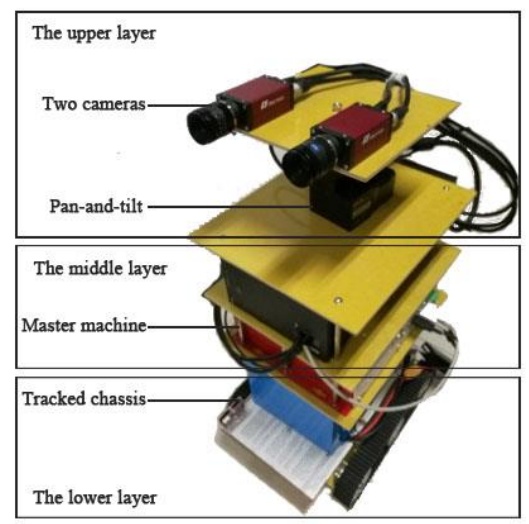

Fig. 1 The unmanned vehicle with three independent hierarchical layers 


\subsection{The ARFM-based VO software system}

The software system contains four modules: camera calibration, ARFM-based 3D reconstruction, position and attitude calculation, BA (Bundle Adjustment) modules. Fig. 2 shows the workflow of the VO software system.

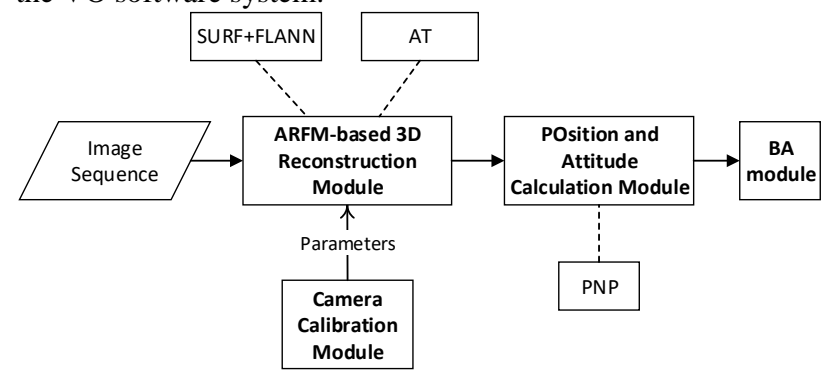

Fig. 2 The workflow of the ARFM-based VO software system.

3.2.1 Camera Calibration Module: Camera calibration is a necessary step in 3D reconstruction in order to extract metric information from 2D images. This paper performs the calibration of binocular stereo measuring system reliably by Zhang's algorithm (Zhang Z, 2002), and gets the extrinsic and intrinsic parameters. In Zhang's algorithm, a $2 \mathrm{D}$ point is denoted by $\left[\begin{array}{ll}x & y\end{array}\right]^{T}$, and a $3 \mathrm{D}$ point is denoted by $\left[\begin{array}{lll}X & Y & Z\end{array}\right]^{T}$. A camera is modeled by the usual pinhole: the relationship between a 3D point and its image projection is given by

$$
s\left[\begin{array}{l}
x \\
y \\
1
\end{array}\right]=A\left[\begin{array}{ll}
R & t
\end{array}\right]\left[\begin{array}{l}
X \\
Y \\
Z \\
1
\end{array}\right] \text {, with } \mathrm{A}=\left[\begin{array}{ccc}
\alpha & c & u_{0} \\
0 & \beta & v_{0} \\
0 & 0 & 1
\end{array}\right]
$$

where $s$ is an arbitrary scale factor; $\left[\begin{array}{ll}R & t\end{array}\right]$, called the extrinsic parameters, is the rotation and translation which relates the world coordinate system to the camera coordinate system; A is called the camera intrinsic matrix, and $\left(\begin{array}{ll}u_{0} & v_{0}\end{array}\right)$ are the coordinates of the principal point, $\alpha$ and $\beta$ the scale factors in image $u$ and $v$ axes, and $c$ the parameter describing the skewness of the two image axes.

3.2.2 ARFM-based 3D Reconstruction Module: In this paper, we propose an ARFM algorithm to guarantee the accuracy and reliability, and adapt to the changed of environment as well. In this algorithm. We use SURF (Speed-up Robust Features) (Bay et al., 2008) to find point correspondences between two images. In order to reduce the computational cost, SURF takes the integral image, which represents the sum of all pixels in the input image I within a rectangular region formed by the origin and $\mathrm{x}$. The integral image $I_{\Sigma}(\mathrm{x})$ at $\left[\begin{array}{ll}x & y\end{array}\right]^{T}$ is

$$
I_{\Sigma}(\mathrm{x})=\sum_{i=0}^{i \leq x} \sum_{j=0}^{j \leq y} I(i, j)
$$

The Hessian-based detectors are scale-invariant, moreover, are more stable and repeatable than their Harris-based counterparts, so Hessian is used to detect feature points.

Given a point $\mathrm{x}=(\mathrm{x}, \mathrm{y})$ in an image $\mathrm{I}$, the Hessian matrix $H(x, \sigma)$ in $x$ at scale $\sigma$ is defined as follows

$$
\mathrm{H}(\mathrm{x}, \sigma)=\left[\begin{array}{ll}
L_{x x}(\mathrm{x}, \sigma) & L_{x y}(\mathrm{x}, \sigma) \\
L_{x y}(\mathrm{x}, \sigma) & L_{y y}(\mathrm{x}, \sigma)
\end{array}\right]
$$

where $L_{x x}(\mathrm{x}, \sigma)$ is the convolution of the Gaussian second order derivative $\frac{\partial^{2}}{\partial_{x^{2}}} g(\sigma)$ with the image I in point $\mathrm{x}$, and similarly for $L_{x y}(\mathrm{x}, \sigma)$ and $L_{y y}(\mathrm{x}, \sigma)$.

SURF used box filter to guarantee scale-invariance and integral image to avoid iterative calculation, so it can extract feature points quickly. According to the neighbourhood of these points, descriptors which are robust to noise, detection displacements and geometric and photometric deformations, are generated. The descriptor is similar to the gradient information extracted by SIFT, but use only 64 dimensions, which reduces the time for feature computation. After that, the descriptor vectors between different images is matched based on Euclidean distance.

But SURF is high error matching, so optimized bothway FLANN (Fast Library for Approximate Nearest Neighbors) (M Muja et al., 2009) is used to match SURF descriptor. Given a set points $\mathrm{P}=\left\{p_{1}, \ldots, p_{n}\right\}$ which is extracted from left image and $\mathrm{Q}=$ $\left\{q_{1}, \ldots, q_{n}\right\}$ which is extracted from right image, and $\operatorname{dist}\left(p_{i}, q_{j}\right)$ which is the Euclidean distance from $p_{i}$ to $q_{j}$. Then the nearest neighbour $\operatorname{dist}\left(p_{i}, q_{j}\right)$ and the second-nearest neighbour $\operatorname{dist}\left(p_{i}, q_{k}\right)$ can be calculated. $\operatorname{dist}\left(p_{i}, q_{j}\right)$ is the optimal value if $\operatorname{dist}\left(p_{i}, q_{j}\right)$ is far less than $\operatorname{dist}\left(p_{i}, q_{k}\right)$ so that $\left(p_{i}, q_{j}\right)$ is a pair of matching points. However, if $\operatorname{dist}\left(p_{i}, q_{j}\right)$ is similar to $\operatorname{dist}\left(p_{i}, q_{k}\right), q_{j}$ and $q_{k}$ is too similar to decide who is the matching point to $p_{i}$, so both points must be abandoned. Only in the condition of $\left(p_{i}, q_{j}\right)$ and $\left(q_{j}, p_{i}\right)$ is the same can be matched. In order to guarantee precision, RANSAC is used to eliminate the mismatched feature points. In this way, the accuracy and reliability requirement can be met. Meanwhile, scale and rotation distortions can be overcame.

Traditional matching method uses a FT (fixed Threshold) algorithm. To adapt the environment variation, we proposed an AT (Adaptive Threshold) algorithm that can automatically adjust the threshold according to the variances of image.

$$
\begin{gathered}
\text { Gray }=\frac{\sum_{i=1}^{M} \sum_{j=1}^{N} G(i, j)}{M \times N} \\
\sigma^{2}=\frac{\sum_{i=1}^{M} \sum_{j=1}^{N}[G(i, j)-\mathrm{Gray}]^{2}}{M \times N}
\end{gathered}
$$

where $\mathrm{G}(i, j)$ are the gray values of current image and Gray is the average gray value of current image, $M$ and $N$ are the height and width of current image. $\sigma^{2}$ is the variances of current image, which can be used to adjust the threshold automatically. And then the $3 \mathrm{D}$ point cloud is generated using the calibration and the feature matching results. The focus length of camera is denoted by $f$, and the baseline is denoted by $T$. The value of parallax is denoted by disp. So the depth of 3D point is calculated by

$$
Z=\frac{T f}{\operatorname{disp}}
$$


3.2.3 Position and Attitude Calculation Module: In this module, the current pose of camera is computed according to the $3 \mathrm{D}$ point cloud generated from $3 \mathrm{D}$ reconstruction module and the current 2D image projection point based on a PNP (PerspectiveN-Point) (Fischler et al., 1981) algorithm. To reduce the accumulative error, this paper uses $3 \mathrm{D}$ points within the FOV (Field of View) from all relevant images to build a local map. As is seen in Fig. 3, the straight lines represent the 3D reconstruction, thus the 3D coordinates can be calculated, and the dotted lines represent the $2 \mathrm{D}$ image point projected on current position.

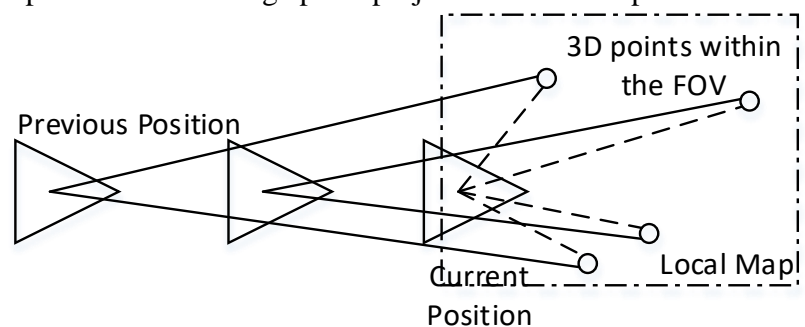

Fig. 3 The principle of position and attitude calculation using local map.

PNP is as follow:

First of all, original $\mathrm{R}$ and $\mathrm{t}$ are calculated from 4 points according to perspective projection model, and the image coordinates $C_{j}^{c}, j=1,2,3,4$ are calculated according to.

$$
\left\{\begin{array}{l}
\sum_{j=1}^{4} \alpha_{i j}\left[f_{x} X_{j}^{c}+Z_{j}^{c}\left(c_{x}-u_{i}\right)\right]=0 \\
\sum_{j=1}^{4} \alpha_{i j}\left[f_{x} Y_{j}^{c}+Z_{j}^{c}\left(c_{y}-v_{i}\right)\right]=0
\end{array}\right.
$$

Then, minimize

$$
\operatorname{Error}(\beta)=\sum_{i, j=1, i<j}^{4}\left|\left\|C_{i}^{c}-C_{j}^{c}\right\|^{2}-\left\|C_{i}^{w}-C_{j}^{w}\right\|^{2}\right|
$$

with the other points according to Gauss-Newton algorithm, thus take $C_{j}^{c}, \mathrm{j}=1,2,3,4$ and $P_{i}^{c}$. Finally, $\mathrm{R}$ and $\mathrm{t}$ can be obtained from $P_{i}^{c}$.

3.2.4 BA Module: BA imposes geometrical constraints over multiple frames, thus providing a global optimal estimate by minimizing the re-projection errors. In this study, global BA (Eudes et al., 2010), which takes the 3D point clouds and all camera poses together at once, is used. The positions of unmanned vehicle and 3D point clouds, including rotation vector and translation vector. And the relationship between positions are noted as edges to build graph. The re-projection errors can be minimized by LM (Levenberg-Marquardt) (Marquardt, 1963) and Gauss-Newton algorithm. The principle of BA is shown in Figure 4.

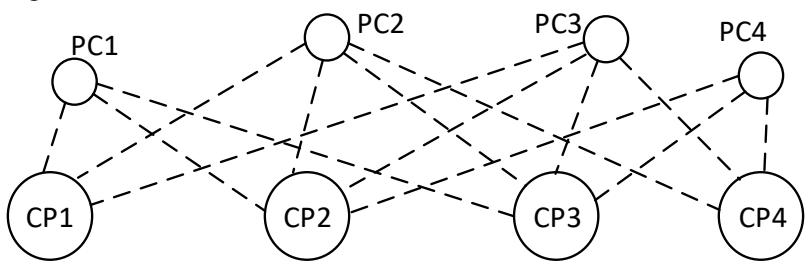

Fig. 4 The principle of BA.

\section{EXPERIMENT}

In this study, the performance of the matching algorithm and the ARFM-based VO software system are tested. First, an algorithm experiment is performed, which proved ARFM is useful to improve the matching precision. Then, two experiments have been conducted to test the VO software. Our platform is tested by the indoors image sequence captured by binocular camera equipped on our platform using internal and external parameters, which are approached by calibration. In the other experiment, the ARFM-based VO software system is tested using an open dataset: KITTI, which are outdoors image sequence captured by binocular camera equipped on moving cars.

\subsection{Experiments}

This experiment section is made up of two parts: the comparison results of matching using our adaptive threshold method: AT and the traditional fixed threshold method: FT, the comparison of different matching strategies.

4.1.1 The comparison results of matching with AT and FT: As is seen in Fig. 5, the number of matching points is increasing and the matching accuracy is reducing with the increasing of threshold value, so it is necessary to take an AT to balance them. The AT of Fig. 6(a) is around 0.375, which is the variance of the image, according to the result shown in Fig. 5(a). Likewise, the AT of Fig.6 (c) is around 0.734.

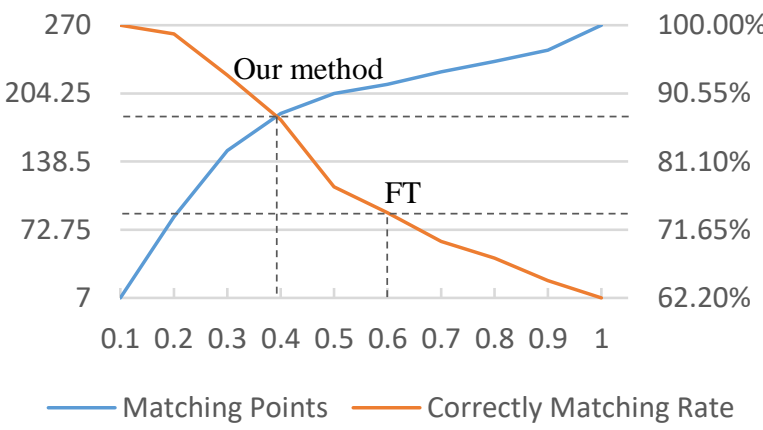

(a)

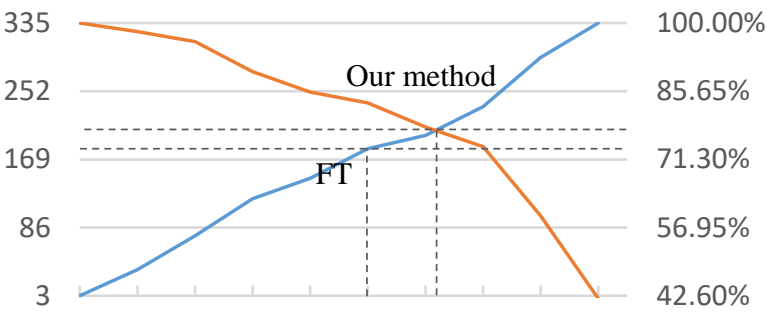

$\begin{array}{lllllllllll}0.1 & 0.2 & 0.3 & 0.4 & 0.5 & 0.6 & 0.7 & 0.8 & 0.9 & 1\end{array}$

Matching Points C_ Correctly Matching Rate

(b)

Fig. 5 The change in matching points and matching accuracy with the increasing of variance. (a) The result of Fig. 6(a). (b) The result of Fig. 6(c)

The traditional algorithm, which sets the threshold value to a FT (fixed threshold), leads to mismatching points or loss of matching points. In the example shown in Fig. 6, the matching result using the AT (0.375) is Fig. 6(a), and the matching result using the FT (0.6) is Fig. 6(b).The comparison between the AT and FT shows that when the threshold is set much larger than the AT, both the matching points and correctly matching rate are reduced obviously. 


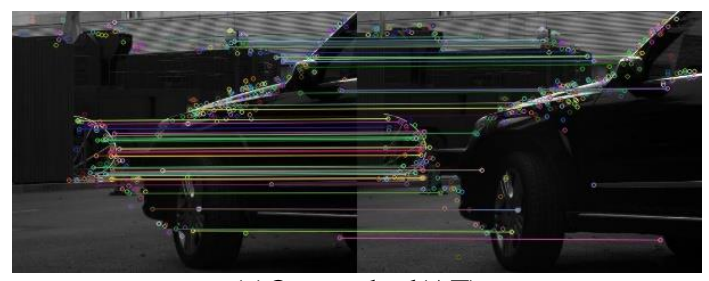

(a)Our method(AT)

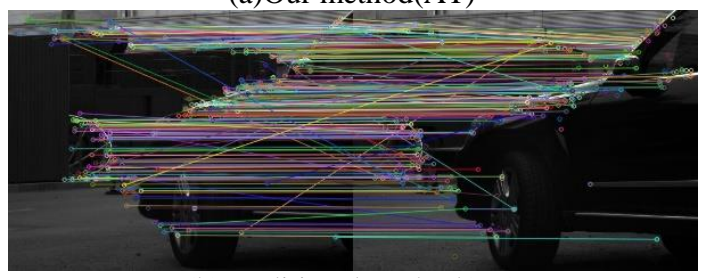

(b)Traditional method(FT)

Fig. 6 The comparison between using the AT and the larger FT. (a) Matching result with AT $=0.375$, the number of matching points is 175 and the correctly matching rate is $87.02 \%$. (b) Matching result with FT $=0.6$, the number of matching points is 83 and the correctly matching rate is $74.28 \%$.

In the example shown in Fig. 7, the adaptive threshold is larger compared with the value in Fig. 6. And the matching result using the adaptive threshold (0.734) is Fig. 7(a), the matching result using the fixed threshold (0.6) is Fig. 7(b). The comparison between the adaptive threshold and fixed threshold shows that when the threshold is set much smaller than the adaptive, the number of correctly matching points is less.

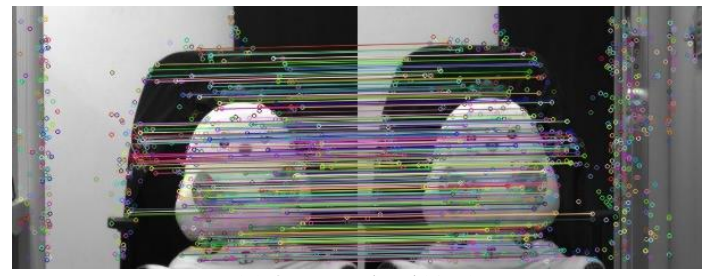

(a)Our method(AT)

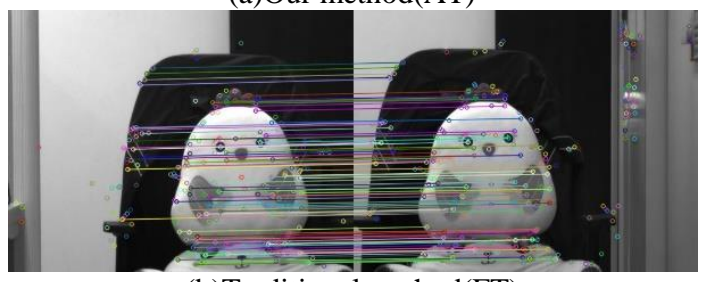

(b)Traditional method(FT)

Fig. 7 The comparison between using the AT and the smaller FT. (a) Matching result with AT $=0.734$, the number of matching points is 212 and the correctly matching rate is $78.24 \%$. (b) Matching result with FT $=0.6$, the number of matching points is 172 and the correctly matching rate is $71.9 \%$.

So, we can draw the conclusion that taking the variance, which is calculated according to the image, as threshold value, contributes to guarantee the maximization of both the matching points and matching accuracy, and set the threshold value as variance of image dynamically is necessary.
4.1.2 The comparison of different matching strategies: As discussed, ARFM is made up of SURF, FLANN, AT and RANSAC. The matching result of four strategies is shown in Fig. 8 , and the statistics is shown in Fig. 9. The number of matching points and correctly matching points is reduced, but the matching rate is increasing with four strategies: SURF, SURF+RANSAC, SURF+FLANN, SURF+FLANN+RANSAC, so ARFM has the highest matching accuracy in these methods.

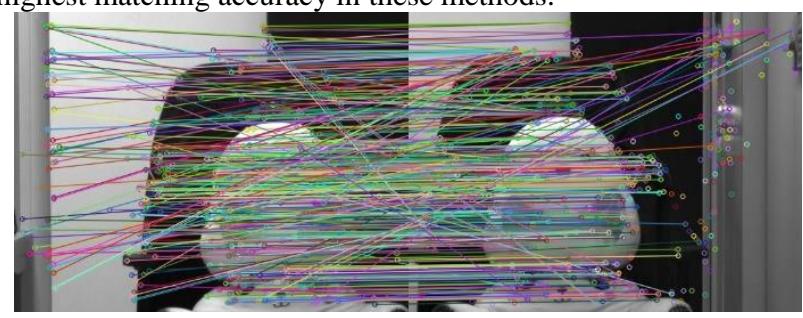

(a) Matching result of SURF

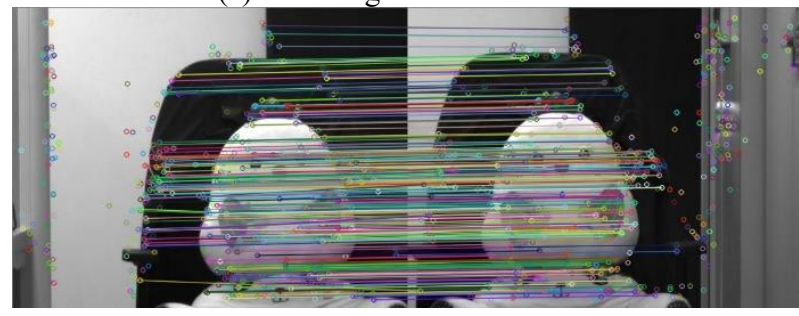

(b) Matching result of SURF+RANSAC

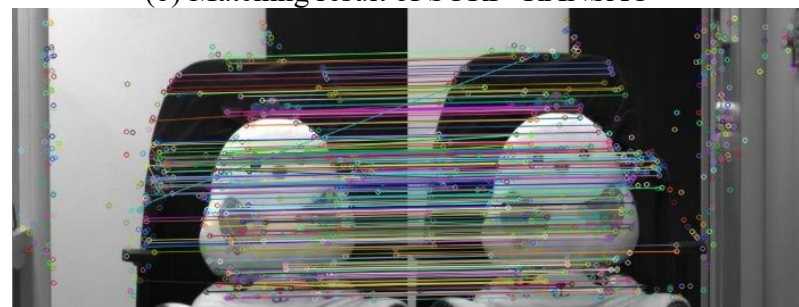

(c) Matching result of SURF+FLANN

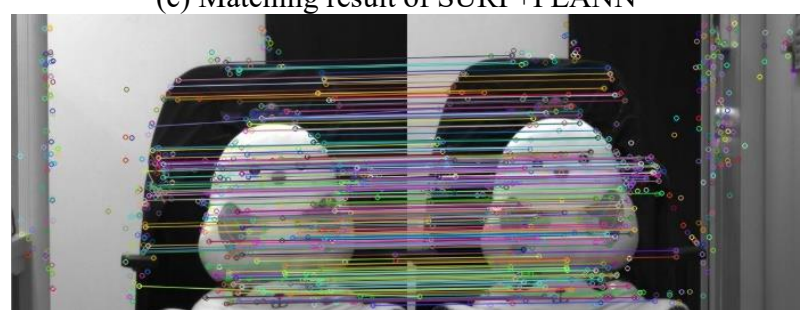

(d) Matching result of SURF+FLANN+RANSAC

Fig. 8 The matching result using four strategies.

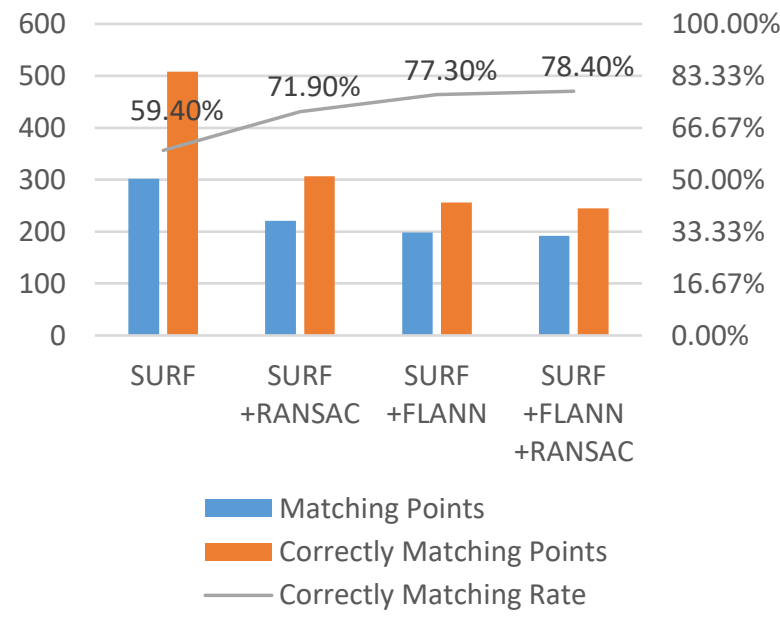

Fig. 9 The statistics of matching result with four strategies. 
Fig. 10 shows the trajectories of four statistics and ground truth, and Fig. 11 shows the translation error and run time of four strategies. From the comparison, we observe that SURF is the worst algorithm not only in translation error but also in run time. SURF+FLANN is of higher precision relativity, while the speed is the highest. The run time of SURF+RANSAC and SURF+FLANN+RANSAC is similar, but the precision of SURF+FLANN+RANSAC is higher. So, if the precision is the most important, SURF+FLANN+RANSAC is the best choice, and if the run time is the most important, SURT+FLANN is the best choice.

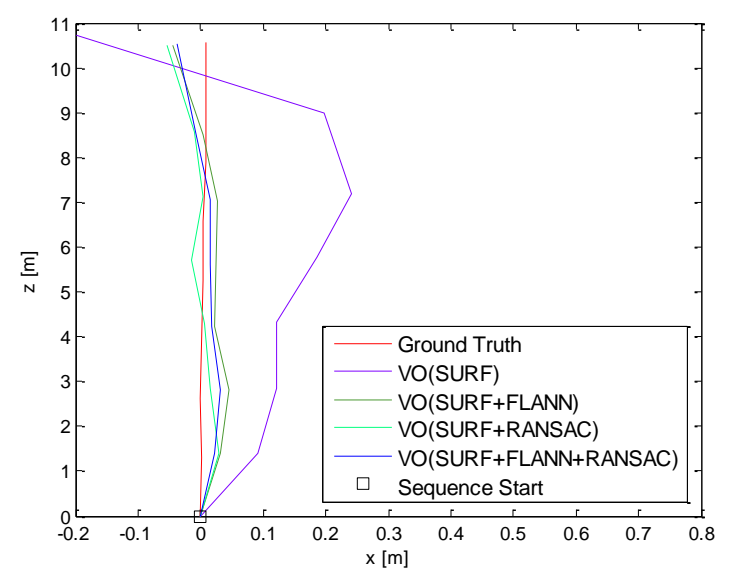

Fig. 10 The trajectories of four methods and ground truth.

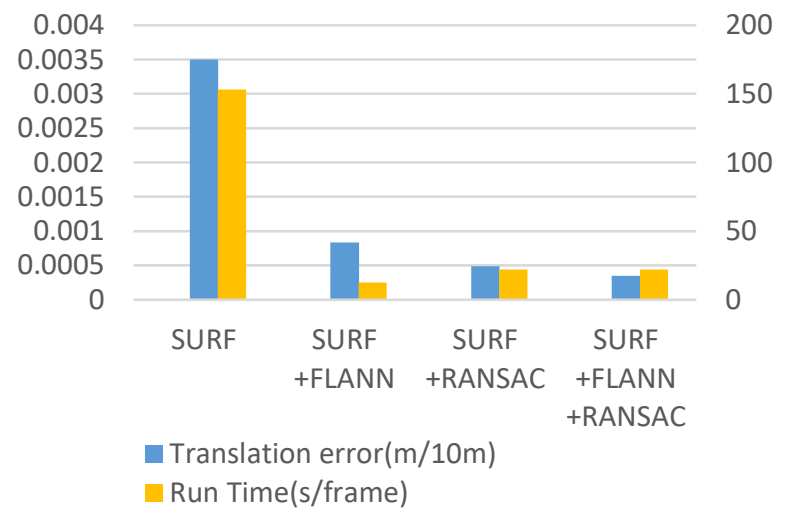

Fig. 11 The translation error and run time of four methods.

\subsection{Indoor VO Experiment}

In this experiment, a specifically designed calibration board is made to calibrate. The board is full of black and white squares whose size is $210 \times 110 \mathrm{~cm}$. Each square is $10 \times 10 \mathrm{~cm}$ so that the calibration board has $20 \times 10$ corners. By using the unmanned vehicle we built, 63 pairs of stereo calibration images were collected, which are shown in Fig.12, and the results of calibration are showed in Fig.13. Fig. 13(a) is a corner detection of the calibration board. Fig. 13(b) shows a rectified result. Meanwhile, internal and external parameters of each camera is obtained.
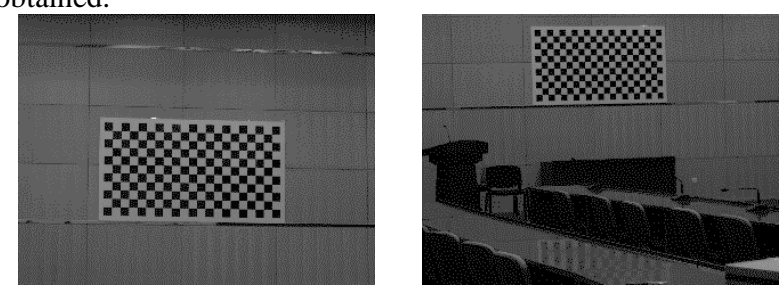

(a)

(b)

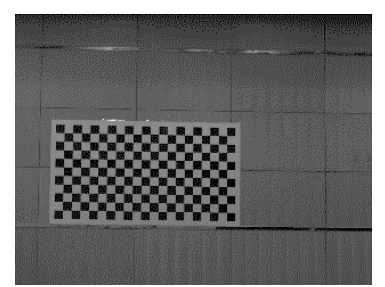

(c)

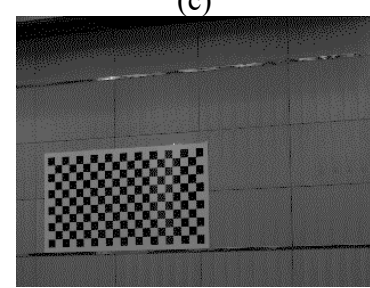

(e)

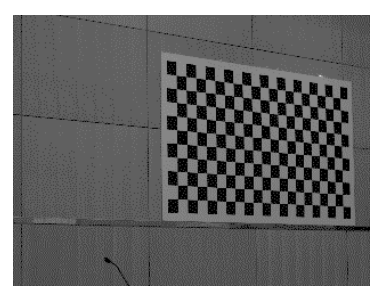

(d)

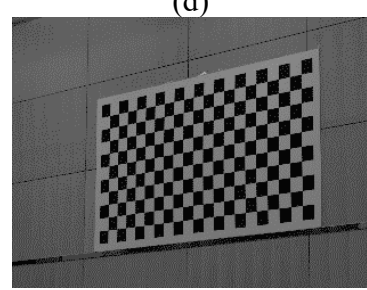

(f)
Fig. 12 Images of the calibration board

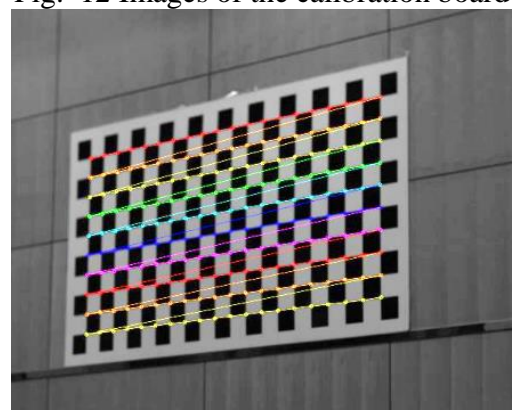

(a) Corner detection.

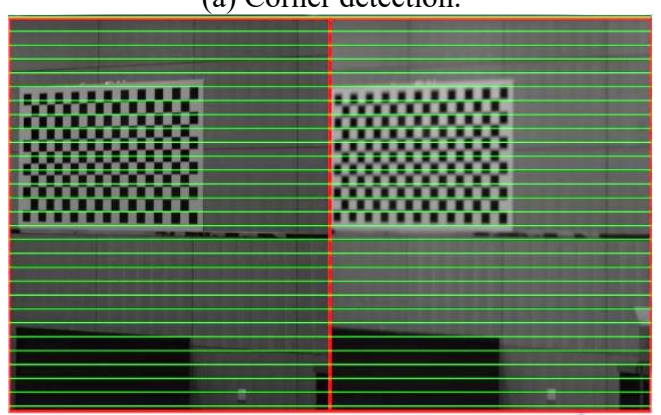

(b) A rectified result.

Fig. 13 The result of stereo calibration.

Then, we tested our platform indoors on 16 December. 2017. In this experiment, an image sequence of 6 pairs with $1624 \times 1234$ resolution was captured. Some of the images are shown in Fig. 14. The experimental results are shown in Fig. 15.

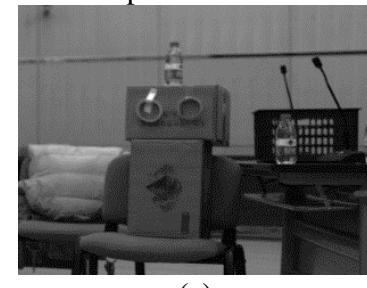

(a)

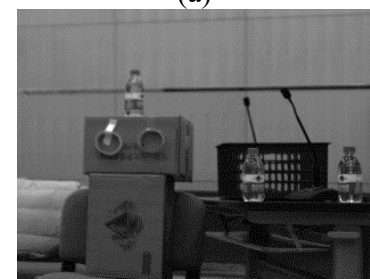

(c)

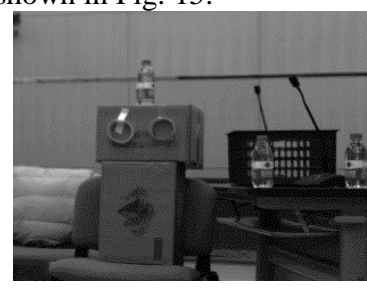

(b)

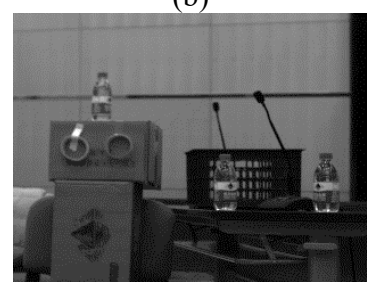

(d) 


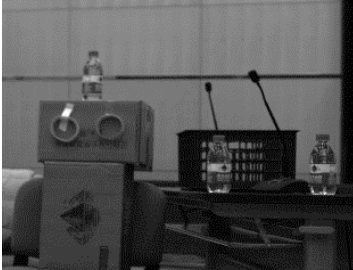

(e)

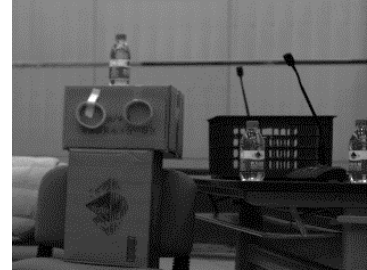

(f)
Fig. 14 Images the unmanned vehicle captured to perform the first experiment.

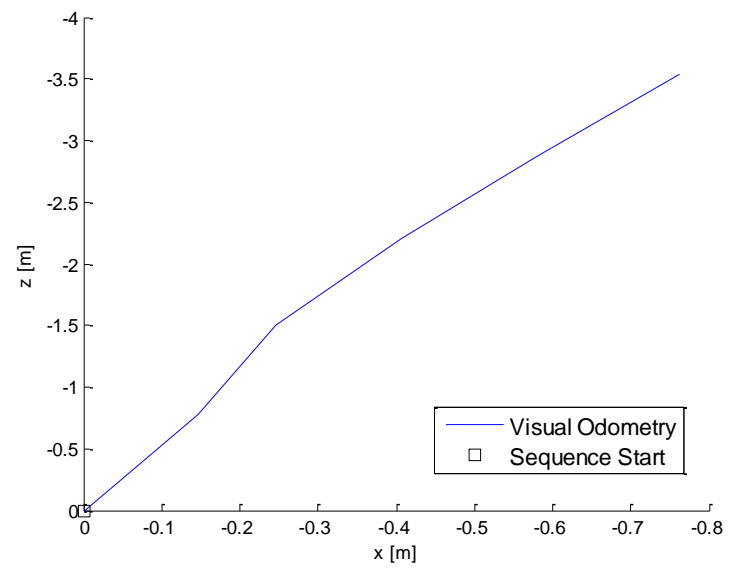

Fig. 15 The result of ARMF-base VO system using the images we captured. In this coordinates, the blue line represents the path calculated by the ARMF-base VO system, and the black point represents the start position.

\subsection{Outdoor VO Experiment}

An image sequence of 100 pairs with $1226 \times 370$ resolution was tested using ARFM-based VO system, some experiment images are shown in Figure 16.

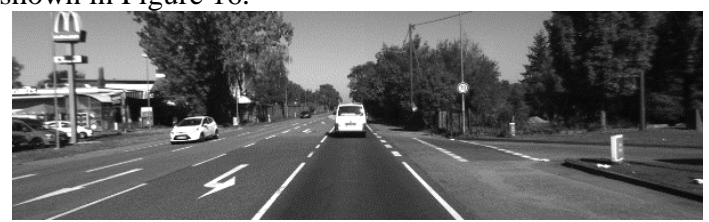

(a)

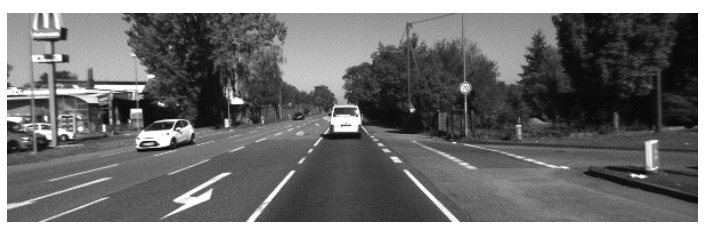

(b)

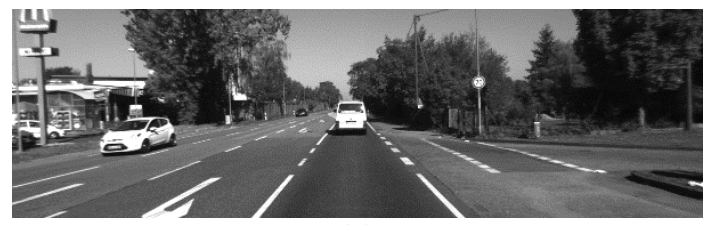

(c)

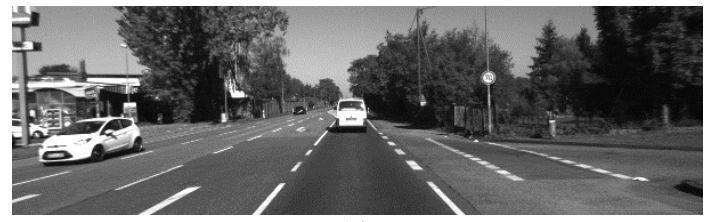

(d)

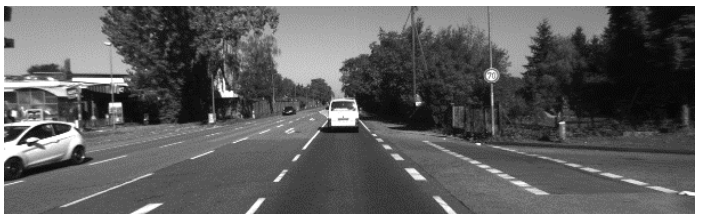

(e)

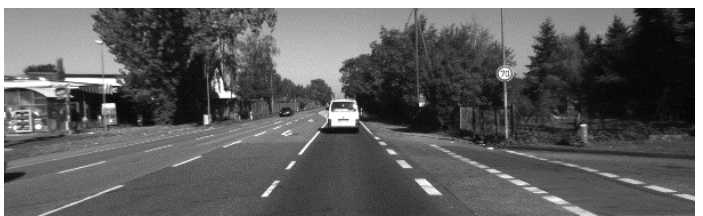

(f)

Fig. 16 Stereo images downloaded from KITTI.

The experimental results are shown in Fig. 17. Fig. 17(a) shows the feature points extracted in this image, and all these points have been reconstruction, so that the blue circle represents the nearest feature point and the red circle represents the farthest one. From the trajectories in Fig. 17(b), it can be seen that the scale propagation error in about 75 meters is about 0.85 meters, but in about 140 meters, it is reduced to 0.3 meters. From the calculations, we conclude that the average translation error is about 0.53 meters per 100 meters. So we can draw the conclusion that ARFM-based VO system contributes to eliminate the accumulative error of translate error, and the accuracy of this platform is $0.53 \%$, which can meet the requirement of planetary exploration. This platform takes $7.25 \mathrm{~s}$ to process a pair of image, so it can location itself per 0.725 meters, which can be used for planetary exploration.

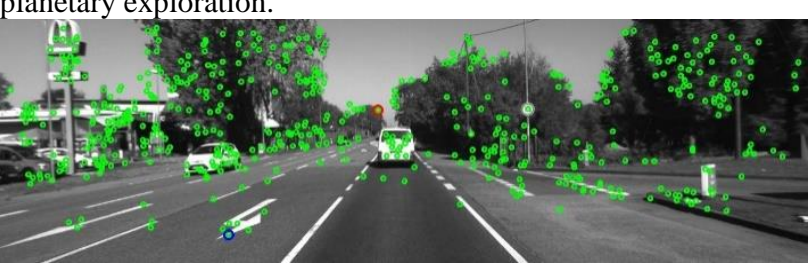

(a)

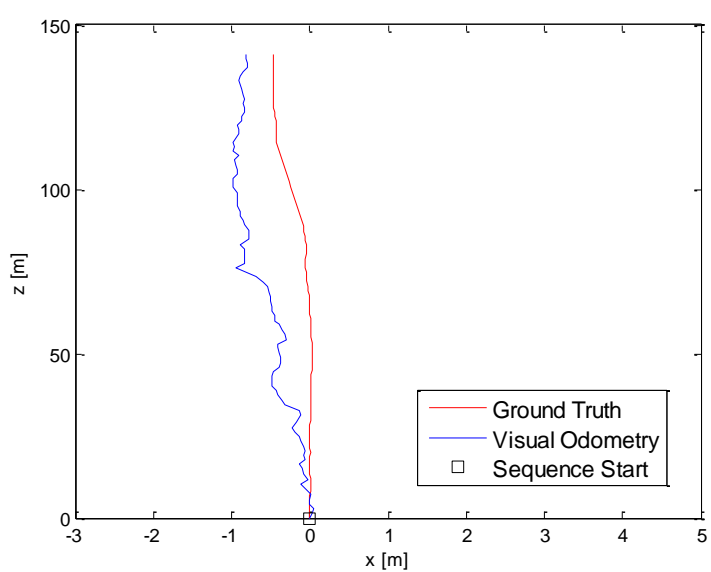

(b)

Fig. 17 The result of ARMF-based VO system using stereo images download from KITTI dataset. (a) An image used to show the feature points. In this image, the green circle represent the feature points which have been matched, the blue circle represents the nearest feature point and the red circle represents the farthest one. (b) The comparison of two trajectories. The red line represents the ground truth, the blue line represents the path calculated by the ARMF-based VO system, and the black point represents the start position. 


\section{CONCLUSION}

In this paper, an autonomous GPS-denied unmanned vehicle platform based on binocular vision has been designed and built for planetary exploration. An ARMF-base VO system containing four modules, has been developed to achieve vision-based GPSdenied autonomous navigation. The algorithm experiment proves that our ARMF is the best choice applied to our unmanned vehicle. Then, experiments using both outdoor images from open dataset and indoor images captured by our vehicle demonstrate that our unmanned vehicle, which combined both hardware and software system, is able to achieve autonomous localization and has the potential for future planetary exploration.

\section{REFERENCES}

Cheng Y, Maimone M, Matthies L. Visual odometry on the Mars Exploration Rovers[C]// IEEE International Conference on Systems, Man and Cybernetics. IEEE, 2006:903-910 Vol. 1.

Sumner D, Mars Science Laboratory Team. Curiosity on Mars: The Latest Results from an Amazing Mission[C]// American Astronomical Society Meeting. American Astronomical Society Meeting Abstracts, 2013.

Zhou J, Xie Y, Zhang Q, et al. Research on mission planning in teleoperation of lunar rovers[J]. Scientia Sinica, 2014, 44(4):441.

Griffiths A D, Coates A J, Jaumann R, et al. Context for the ESA ExoMars rover: the Panoramic Camera (PanCam) instrument[J]. International Journal of Astrobiology, 2006, 5(3):269-275.

Stephens M. A Combined Comer and Edge Detector[J]. 1988.

Eudes A, Naudet-Collette S, Lhuillier M, et al. Weighted Local Bundle Adjustment and Application to Odometry and Visual SLAM Fusion[C]// British Machine Vision Conference, BMVC 2010, Aberystwyth, UK, August 31 - September 3, 2010. Proceedings. DBLP, 2010:1-10.

Lowe D G. Object Recognition from Local Scale-Invariant Features[C]// Proc. IEEE International Conference on Computer Vision. 1999:1150.

Zhang Z. Flexible Camera Calibration by Viewing a Plane from Unknown Orientations[C]// The Proceedings of the Seventh IEEE International Conference on Computer Vision. IEEE, 2002:666-673 vol.1.

Bay H, Ess A, Tuytelaars T, et al. Speeded-Up Robust Features (SURF)[J]. Computer Vision \& Image Understanding, 2008, 110(3):346-359.

Muja M. FLANN -Fast Library for Approximate Nearest Neighbors User Manual[J]. 2009.

Bolles R C, Fischler M A. RANSAC-BASED APPROACH TO MODEL FITTING AND ITS APPLICATION TO FINDING CYLINDERS IN RANGE DATA[C]// International Joint Conference on Artificial Intelligence. 1981.

Fischler M A, Bolles R C. Random sample consensus: a paradigm for model fitting with applications to image analysis and automated cartography[M]. ACM, 1981. 